\title{
miR-744-5p Inhibits Multiple Myeloma Proliferation, Epithelial Mesenchymal Transformation and Glycolysis by Targeting SOXI2/Wnt/ $\beta$-Catenin Signaling
}

This article was published in the following Dove Press journal: OncoTargets and Therapy

\author{
Bingling Guo ${ }^{1, *}$ \\ Chunyan Xiao',* \\ Yumin Liu ${ }^{2}$ \\ Ning Zhang ${ }^{3}$ \\ $\mathrm{HaO} \mathrm{Bai}^{4}$ \\ Tao Yang' \\ Ying Xiang' \\ Yingyu Nan' \\ Qiying $\mathrm{Li}^{\prime}$ \\ Wenjun Zhang' \\ Dehong Huang' \\ 'Department of Hematology and \\ Oncology, Chongqing University Cancer \\ Hospital, Chongqing, People's Republic of \\ China; ${ }^{2}$ Medical Records Management \\ Division, Chongqing University Cancer \\ Hospital, Chongqing, People's Republic of \\ China; ${ }^{3}$ Intensive Care Unit, Chongqing \\ University Cancer Hospital, Chongqing, \\ People's Republic of China; ${ }^{4}$ Pharmacy \\ Services, Chongqing University Cancer \\ Hospital, Chongqing, People's Republic of \\ China
}

*These authors contributed equally to this work

Correspondence: Dehong Huang Department of Hematology and

Oncology, Chongqing University Cancer Hospital, No. I8I Hanyu Road,

Chongqing, People's Republic of China

$\mathrm{Tel}+86$ 23-653il34I

Email dehong_h012436@163.com

\begin{abstract}
Purpose: This study investigated the function and molecular mechanisms of miR-744-5p in multiple myeloma (MM).

Methods: miR-744-5p and SRY-related high-mobility-group box 12 (SOX12) expression in clinical tissues and MM cells was monitored by quantitative real-time polymerase chain reactions and Western blot. miR-744-5p expression in MM cells was regulated by transfection. Cell proliferation was researched by cell counting kit-8 assay and plate clone formation experiment. Transwell experiment was utilized for migration and invasion detection. Glycolysis test was conducted for the detection of glucose uptake and lactate production of MM cells. The relationship between miR-744-5p and SOX12 was determined by dual-luciferase reporter gene assay and RNA pull-down experiment. In vivo experiment was conducted using nude mice.
\end{abstract}

Results: miR-744-5p expression was reduced in MM patients $(P<0.01)$. Low miR-744-5p expression was associated with lower 60 -month survival in MM patients $(P=0.0402)$. miR$744-5 p$ overexpression inhibited MM cells proliferation, invasion, migration, glucose uptake, lactate production, and epithelial mesenchymal transformation (EMT) $(P<0.01)$. miR-744-5p directly inhibited SOX12 expression. miR-744-5p silencing promoted MM cells proliferation, invasion, migration, glucose uptake, lactate production, and EMT by elevating SOX12 $(P<0.01)$. miR-744-5p inhibited the growth of MM xenograft tumors in vivo $(P<0.001)$.

Conclusion: miR-744-5p inhibits MM cells proliferation, invasion, migration, EMT, and glycolysis by targeting SOX12/Wnt/ $\beta$-catenin.

Keywords: MM, miR-744-5p, SOX12, EMT, glycolysis

\section{Introduction}

Multiple myeloma (MM) is a fatal hematologic malignancy, accounting for approximately $10 \%$ of hematological tumor diseases. ${ }^{1} \mathrm{MM}$ occurs in the bone marrow and about 80,000 patients are newly diagnosed with $\mathrm{MM}$ annually. ${ }^{2}$ In spite of advances in MM diagnosis and treatment, such as chemotherapy and monoclonal antibodies therapy, a majority of MM cases suffered a poor prognosis due to the seriousrecurrence rate. ${ }^{2,3}$ Furthermore, the uncontrolled MM cells growth makes the disease still incurable. ${ }^{4}$ Abnormal changes of cell genetics are important causes of MM. However, the pathogenesis and detailed underlying mechanism of MM has not been clearly clarified. Therefore, it is of urgent need to explore the occurrence and development mechanism of MM at the molecular level. 
microRNAs are considered as important markers and targets for tumor diagnosis and treatment. They have gained great attention by lots of scientific researchers. miRNAs are highly conserved endogenous small noncoding RNAs with a length of approximately 22 nucleotides. ${ }^{5}$ They playa pivotal role in tumorigenesis and development of human tumors. The carcinogenic or anti-cancer effects of miRNAs have been reported in the pathogenesis of various malignant tumors. ${ }^{6,7}$ Among numerous miRNAs, miR-744-5p has been found to be abnormally expressed in some solid tumors. Kleemann et $\mathrm{al}^{8}$ revealed that the expression of miR-744-5p was declined in ovarian cancer. Patients with high miR-744$5 p$ expression exhibited a longer median disease-free survival. Simultaneously, miR-744-5p up-regulation activated the intrinsic apoptotic pathway, thereby resulting in the death of ovarian cancer cells. Besides, researchers demonstrated that miR-744-5p could attenuate non-small cell lung cancer cells proliferation and invasion via targeting paired box $2 .{ }^{9}$ In addition, Sui et al ${ }^{10}$ revealed that miR744-5p was participated in regulating lung adenocarcinoma progression via being sponged by long noncoding RNA MAFG-AS1. However, the molecular mechanism of miR-744-5p in regulating MM has not yet been elucidated in detail. SRY-related high-mobility-group box 12 (SOX12) was previously reported to be up-regulated in MM. The elevated SOX12 expression exacerbated the growth of MM cells both in vitro and in vivo. The mechanism was by enhancing the $\mathrm{Wnt} / \beta$-catenin signaling pathway. However, whether SOX12 was regulated by miRNAs in MM was rarely studied. ${ }^{4}$ Thus, this study evaluated the expression and function of miR-744-5p in MM. The interaction between miR-744-5p and SOX12/ $\mathrm{Wnt} / \beta$-catenin signaling pathway was further researched. The findings of this research will provide a novel target and more reliable theoretical basis for the treatment of MM.

\section{Methods}

\section{Patients and Tissue Samples}

Bone marrow tissues were collected from 73 patients with MM using bone marrow aspiration method. The $73 \mathrm{MM}$ cases were first diagnosed with MM from June 2011 to November 2014 in Chongqing University Cancer Hospital. In addition, bone marrow tissues from 73 healthy participants were obtained as control. The 73 healthy participants underwent bone marrow aspiration in our hospital during the same period, and were finally diagnosed with normal bone marrow function. All of the bone marrow tissues were not selected for MM positive cells. Therefore, a total of 146 bone marrow tissue samples were collected in this research (healthy 73 and MM 73). The expression of miR-744-5p in these clinical tissues was determined by quantitative real-time polymerase chain reactions (qRTPCR). A 60-month follow-up was performed in 73 patients with MM. Patients 60-month survival was analyzed by Kaplan-Meier Survival Curve. This study has been approved by the ethics committee of Chongqing University Cancer Hospital in compliance with the Declaration of Helsinki. Written informed consent has been signed by all participants.

\section{Cell Lines}

Normal plasma cells (nPCs) and MM cells (NCI-H929, KM3, H929, U1996, and U266) were obtained commercially from the Institute of Biochemistry and Cell Biology of the Chinese Academy of Sciences (Shanghai, China). RPMI 1640 medium containing 10\% fetal bovine serum (FBS) (Solarbio, Beijing, China) and 1\% penicillin/streptomycin (Solarbio, Beijing, China) was used for cells culture. Cells were kept in a sterile incubator with $95 \%$ humidity at $37^{\circ} \mathrm{C}, 5 \% \mathrm{CO}_{2}$. The RPMI 1640 medium was changed every 3 days.

\section{Transfection}

miR-744-5p mimic, miR-744-5p mimic negative control (NC), miR-744-5p inhibitor, miR-744-5p inhibitor NC and SOX12 small interfering RNA (siRNA) were designed and synthesized by Genepharma (Shanghai, China). U1996 and NCI-H929 cells were cultured in 6-well plates with serum-free RPMI 1640 medium. Then these cells were transfected by miR-744-5p mimic (miR-744-5p mimic group) or miR-744-5p mimic NC (miR-NC group). miR$744-5 p$ inhibitor (miR-744-5p inhibitor group) or miR744-5p inhibitor NC (NC-inh group) was utilized for the transfection of U1996 cells. Co-transfection was performed on U1996 cells using both miR-744-5p inhibitor and SOX12 siRNA (miR-744-5p inhibitor + siSOX12 group). Lipofectamine 2000 reagent (Thermo Fisher Scientific, Waltham, MA, USA) was responsible for the transfection strictly according to the manual. Cells were transfected for 8 hours at $37^{\circ} \mathrm{C}, 5 \% \mathrm{CO}_{2}$. After the residual liquid was discarded, RPMI 1640 medium with $10 \%$ FBS was added into each well. Cells were continuously kept in the incubator with $95 \%$ humidity at $37^{\circ} \mathrm{C}, 5 \% \mathrm{CO}_{2}$. 


\section{Cell Counting Kit-8 (CCK-8) Assay}

U1996 and NCI-H929 cells were collected and plated in 96well plates with $1 * 10^{4}$ cells per well. RPMI 1640 medium with $10 \%$ FBS was added into each well with a volume of $100 \mu \mathrm{L}$. After 24,48 , and 72 hours culture at $37^{\circ} \mathrm{C}, 5 \% \mathrm{CO}_{2}$, $10 \mu \mathrm{L}$ of CCK-8 reagent (Solarbio, Beijing, China) was added into each well to incubate cells for 2 hours at $37^{\circ} \mathrm{C}$. Using a microplate reader (Bio-Rad, Hercules, CA, USA), the optical density (OD) value at 450 wavelength was measured. The experiment was repeated three times.

\section{Plate Clone Formation Experiment}

U1996 and NCI-H929 cells were inoculated in 6-well plates with $1 \mathrm{~mL}$ of RPMI 1640 medium (10\% FBS). In total, 1,000 cells were plated in each well. Cells were kept in the incubator at $37^{\circ} \mathrm{C}, 5 \% \mathrm{CO}_{2}$ for 14 days. The medium in each well was changed every 3 days. On the 14 th day, $4 \%$ paraformaldehyde was utilized for the fixation of cells. Then $0.1 \%$ crystal violet was applied for the staining of cells. Cells in each well were observed under an inverted microscope (Olympus, Tokyo, Japan). The colony number was counted. A colony with more than 50 cells was considered as one colony formation. The experiment was repeated three times.

\section{Transwell Experiment}

A total of $1 * 10^{3}$ U1996 or NCI-H929 cells were suspended in $500 \mu \mathrm{L}$ of serum-free RPMI 1640 medium. Cells were then added into 6-well Transwell insert (pore size of $8 \mu \mathrm{m}$ ). Matrigel $(100 \mu \mathrm{L})$ was pre-spread onto the upper chamber. All the Transwell inserts were inserted into 6-well plates containing $600 \mu \mathrm{L}$ of RPMI 1640 medium (with $10 \%$ FBS). Cells were maintained at $37^{\circ} \mathrm{C}, 5 \% \mathrm{CO}_{2}$ for 24 hours. The non-invading cells that attached on the upper chamber were removed using cotton swab. The invading cells were attached on the lower chamber. These cells were experienced with fixation by $4 \%$ paraformaldehyde and staining by $0.1 \%$ crystal violet. The number of invading cells was counted under a microscope in five random nonoverlapping fields. Cells migration was detected as above, except that the upper chamber was not coating with Matrigel. The experiment was repeated three times.

\section{Glucose Uptake and Lactate Production Detection}

After 48 hours post-transfection, U1996 and NCI-H929 cells were collected and were lysed using lysis buffer. After 10 minutes centrifugation at $3,000 \mathrm{rmp} / \mathrm{min}$, the supernatant was obtained. The glucose uptake and lactate production of U1996 and NCI-H929 cells was measured by detecting the level of glucose and lactate in the supernatant. The glucose uptake was detected using glucose assay kit (BioVision, Mountain View, USA) and lactate production was measured using lactate assay kit (BioVision, Mountain View, USA), strictly according to the manuals. The glucose uptake and lactate production was normalized to the protein concentration. The experiment was repeated three times.

\section{Dual-Luciferase Reporter Gene Assay}

Two online databases (Targetscan and Starbase) indicated that SOX12 possessed the binding sites for miR-744-5p in the $3^{\prime}$-UTR area. According to this result, the wild type (WT) and mutant type (Mut) sequences of SOX12-3'-UTR were designed and synthesized by Genepharma (Shanghai, China). The sequences were amplified by PCR and then were loaded onto the psi-CHECK-2 luciferase reporter vector (Promega, Wisconsin, WI, USA). U1996 cells of miR-744-5p mimic group, miR-NC group, miR-744-5p inhibitor group, and NC-inh group were cultured in 6-well plates with serum-free RPMI 1640 medium. psiCHECK-2 luciferase reporter vector loaded with SOX123'-UTR-WT sequence or SOX12-3'-UTR-Mut sequence was used to cotransfect U1996 cells of the four groups. Lipofectamine 2000 reagent was applied for transfection. At 48 hours post-transfection, the Dual-Luciferase Reporter Assay (Promega, Madison, WI, USA) was performed to detect the luciferase activity of cells in each group. The experiment was repeated three times.

\section{RNA Pull-Down Experiment}

RNA pull-down experiment was performed using U1996 cells. Briefly, the biotinylated-SOX12-sense, -SOX12antisense and -SOX12-NC were designed and synthesized by Genepharma (Shanghai, China). U1996 cells were transfected by these probes for 48 hours. After that, cells were lysed for 30 minutes with cell lysis buffer at $4{ }^{\circ} \mathrm{C}$ (Boster, Wuhan, China). The supernatant of cell lysates was obtained by 10 minutes centrifugation at $4{ }^{\circ} \mathrm{C}$, $12,000 \times \mathrm{g}$. A total of $50 \mu \mathrm{L}$ of the samples were aliquoted for input. Then Dynabeads M-280 Streptavidin (Invitrogen Life Technologies, Carlsbad, CA, USA) was used to incubate with the remaining lysates in accordance with the manuals. After being treated with RNase-free solutions, the beads (Invitrogen Life Technologies, Carlsbad, CA, USA) were subjected to 10 minutes incubation with 
equal volume of biotinylated SOX12 at room temperature on a rotator. Thereafter, ethylene diamine tetraacetic acid (EDTA) and 95\% formamide was used to incubate the beads for 5 minutes. Finally, the eluted miR-744-5p was quantified through qRT-PCR. The experiment was repeated three times.

\section{qRT-PCR}

Tissues and cells were treated with Trizol reagent to obtain total RNA. The concentration of total RNA was determined by NanoDrop 2000 spectrophotometer (Thermo Fisher Scientific, Waltham, MA, USA). A total of $1 \mu \mathrm{g}$ RNA sample was subjected to reverse transcription reaction using PrimeScript ${ }^{\mathrm{TM}}$ RT reagent kit (Takara, Shiga, Japan). The reverse transcription reaction of miR-744-5p was performed using a Transcriptor First Strand cDNA Synthesis kit (Roche Diagnostics, Basel, Switzerland) for the synthesis of miR-744-5p cDNA. qPCR was then performed using the synthesized cDNA by an SYBR Premix Ex Taq Kit (Takara, Shiga, Japan) according to the following temperature protocol: $95^{\circ} \mathrm{C}$ for 10 minutes, followed by 40 cycles of $95^{\circ} \mathrm{C}$ for 15 seconds, $60^{\circ} \mathrm{C}$ for 30 seconds and $72^{\circ} \mathrm{C}$ for 60 seconds. Glyceraldehyde 3-phosphate dehydrogenase (GAPDH) was used as the internal control for SOX12 and U6 was responsible for the internal control of miR-744-5p. The primers used were as follows: miR-7445p, forward, 5'-AATGCGGGGCTAGGGCTA-3', reverse, 5'-GTGCAGGGTCCGAGGT-3'. U6, forward-5'-CTC GCTTCGGCAGCACA-3', reverse, 5'-AACGCTTCA CGAATTTGCGT-3'. SOX12, forward, 5'-AGCACC CGTGTGACTCTTTCC-3', reverse, 5'-AGCAGAACCA AGCCCTGTCTC-3'. GAPDH, forward, 5'CACCCACTCСТCCACCTTTG-3', reverse, 5'CCACCACCCTGTTGCTGTAG-3'. The relative expression of miR-744-5p and SOX12 mRNA was calculated by $2^{-\Delta \Delta C T}$ method. The experiment was repeated three times.

\section{Western Blot}

Cells were harvested and incubated with lysis buffer for 15 minutes on ice. Total proteins in the supernatant were obtained through 10 minute centrifugation at 12,000 rpm, $4^{\circ} \mathrm{C}$. The concentration of total proteins was determined by BCA Protein Assay kit (Pierce Biotechnology, Rockford, IL, USA). The separation of proteins was performed via sodium dodecyl sulfate polyacrylamide gel electrophoresis (SDS-PAGE). Thereafter, proteins were transferred onto a polyvinylidene fluoride (PVDF) membrane and then incubated with 5\% skimmed milk for 1 hour at room temperature. In a $4^{\circ} \mathrm{C}$ refrigerator, the PVDF membrane was incubated with primary antibodies overnight. Primary antibodies used in this research were as follows: rabbit anti-SOX12, -E-cadherin, -N-cadherin, and -Slug (1:1,000, Abcam, Cambridge, UK), mouse antiMMP-2 and -MMP-9 (1:1,000, Santa-Cruz Biotechnology, Santa Cruz, CA, USA), rabbit anti-active $\beta$-catenin, and mouse anti-GAPDH (1:1,000, Cell Signaling Technology, Danvers, MA, USA). After three times washing with Trisbuffered saline/0.1\% Tween (TBST), horseradish peroxidase (HRP) conjugated secondary antibodies (1:5,000, Boster, Wuhan, China) were used to incubate the PVDF membrane for 1 hour at room temperature. Enhanced chemiluminescence (ECL) reagent was added onto the PVDF membrane to visualize protein bands. The gray density value of protein bands was determined by ImagePro Plus 6.0. The experiment was repeated three times.

\section{Animal in vivo Experiment}

Xenograft tumor experiment was conducted with nude mice ( 5 weeks old, $n=6$ ). All nude mice were commercially provided by Shanghai Experimental Animal Center, Chinese Academy of Sciences (Shanghai, China). Animal experiments have been approved by the Animal Ethics Committee of Chongqing University Cancer Hospital, and were performed in accordance with relevant guidelines and regulations of the Animal Ethics Committee of Chongqing University Cancer Hospital.

U1996 cells of the miR-NC group and miR-744-5p group were collected. Phosphate buffer solution (PBS) was used to disperse cells to a density of $1 * 10^{7}$ cells $/ \mathrm{mL}$. The six nude mice were kept for 2 weeks with free access to food and water. Then three nude mice were randomly selected and subcutaneously injected with cell suspension $(100 \mu \mathrm{L})$ of miR-NC group. The injection site was located in the center of the back. At the same site, the other three nude mice were subcutaneously injected with cell suspension $(100 \mu \mathrm{L})$ of the miR-744-5p group. All nude mice were kept individually in cages with free access to water and food. Notably, after injection of the transfected cells, nude mice were subcutaneously injected with $30 \mu \mathrm{g}$ of Lipofectamine 2000-encapsulated miR-744-5p mimic or miR-744-5p mimic NC (30 $\mu \mathrm{g})$ every 3 days. ${ }^{11}$ The xenograft tumor size was measured every 7 days with a vernier caliper. The volume of xenograft tumor was calculated with the following formula: tumor volume=width ${ }^{2} *$ length ${ }^{*} 0.5$. On the 28th day post-inoculation, all mice were sacrificed by rapid neck dislocation. Xenograft tumors were stripped 
and weighed. The expression of miR-744-5p and SOX12 in xenograft tumors was investigated by qRT-PCR.

\section{Statistical Analysis}

All results were obtained from at least three independent repeated trials. Data were presented in the form of mean \pm standard deviation. Data were analyzed by SPSS 19 software (SPSS Inc., Chicago, IL, USA) and GraphPad Prism (version 6, La Jolla, CA, USA). Student's $t$-test and one-way analysis of variance were responsible for the comparison between two groups and at least three groups. Pearson's correlation analysis was applied for the correlation analysis between miR-744-5p and SOX12 expression. The threshold for statistically significant differences was set as $P<0.05$.

\section{Results}

\section{The Expression of miR-744-5p Was Reduced in MM Patients and Cells}

In this research, a total of 146 bone marrow tissue samples were collected (healthy 73 and MM 73). Results from qRTPCR revealed that, in comparison with normal bone marrow tissues, the expression of miR-744-5p was distinctly reduced in bone marrow tissues of MM patients $(P<0.01)$ (Figure 1A). These MM patients were followed up for 60 months. With the average value (0.669) of miR-744-5p expression level as the cut-off value, patients were divided into two groups (High miR-744-5p expression group and Low miR-744-5p expression group). Obviously, MM patients with low miR-744-5p expression had much lower 60-month survival than those with high miR-744-5p expression ( $P=0.0402$ ) (Figure 1B). In vitro studies showed that miR-744-5p was seriously downregulated in MM cells (NCI-H929, KM3, H929, U1996, and
U266) than that in normal plasma cells (nPCs) $(P<0.01$ or $P<0.001$ ) (Figure 1C). Notably, U1996 and NCI-H929 cells exhibited lower miR-744-5p expression than the other three MM cell lines. Thus, U1996 and NCI-H929 cells were used in the following experiments.

\section{miR-744-5p Inhibited MM Cells Proliferation, Invasion, Migration, and Glycolysis}

qRT-PCR was performed to research the transfection efficiency of U1996 and NCI-H929 cells. As shown in Figure 2A, relative to miR-NC group, significantly higher miR744-5p expression was occurred in U1996 and NCI-H929 cells of the miR-744-5p mimic group $(P<0.01)$. This result illustrated that U1996 and NCI-H929 cells were successfully transfected. Next, CCK-8 assay and plate clone formation experiment were conduced to detect the effect of miR-744-5p on U1996 and NCI-H929 cells proliferation. Compared to the miR-NC group, prominently lower OD450 value and colony number was found in U1996 and NCI-H929 cells of miR-744-5p mimic group $(P<0.01)$ (Figure $2 \mathrm{~B}$ and $\mathrm{C})$. Transwell experiment indicated that the invasion and migration cell number were both remarkably decreased in miR-744-5p mimic group when compared with the miR-NC group $(P<0.01)$ (Figure 2D). In addition, U1996 and NCI-H929 cells of the miR-744-5p mimic group exhibited markedly lower glucose uptake and lactate production than that of miRNC group $(P<0.01)$ (Figure 2E). According to Western blot, seriously up-regulation of E-cadherin protein and down-regulation of N-cadherin, Slug, MMP-2, MMP-9, and active $\beta$-catenin proteins was observed in U1996 and
A

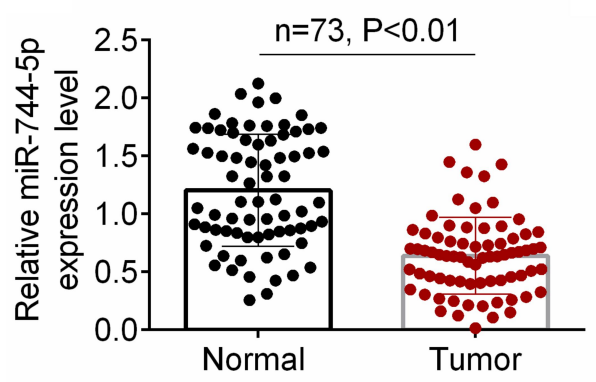

B

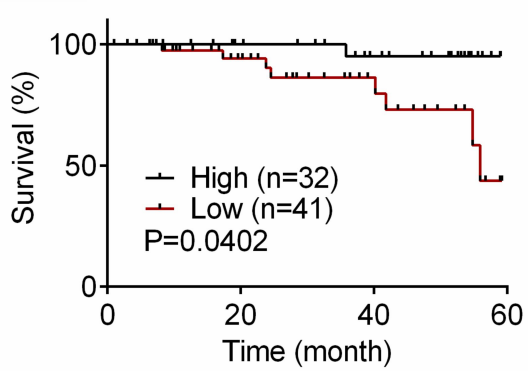

C

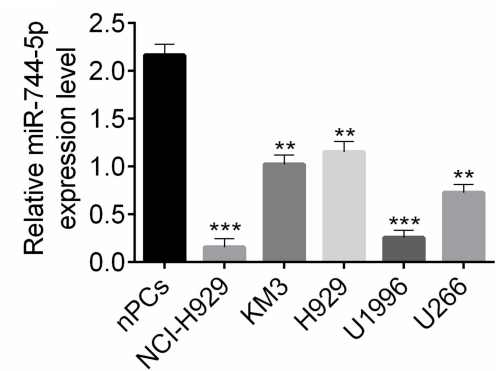

Figure I The expression of miR-744-5p was reduced in MM patients and cells. (A) qRT-PCR revealed that miR-744-5p expression was distinctly down-regulated in bone marrow tissues of MM patients than that in normal bone marrow tissues. A total of 146 bone marrow tissue samples were collected (healthy 73 and $M M$ M 73 ). miR-744-5p expression was normalized to U6. (B) MM patients with low miR-744-5p expression had much lower 60-month survival than those with high miR-744-5p expression. The average value (0.669) of miR-744-5p expression level was as the cut-off value. (C) miR-744-5p was seriously down-regulated in MM cells (NCl-H929, KM3, H929, UI996, and U266) than in normal plasma cells (nPCs). miR-744-5p expression was normalized to U6. $* * P<0.0 \mathrm{I}$ or $* * * P<0.00 \mathrm{I}$ when compared with miR-744-5p expression in $\mathrm{nPCs}$. Abbreviations: MM, multiple myeloma; qRT-PCR, quantitative real-time polymerase chain reactions. 

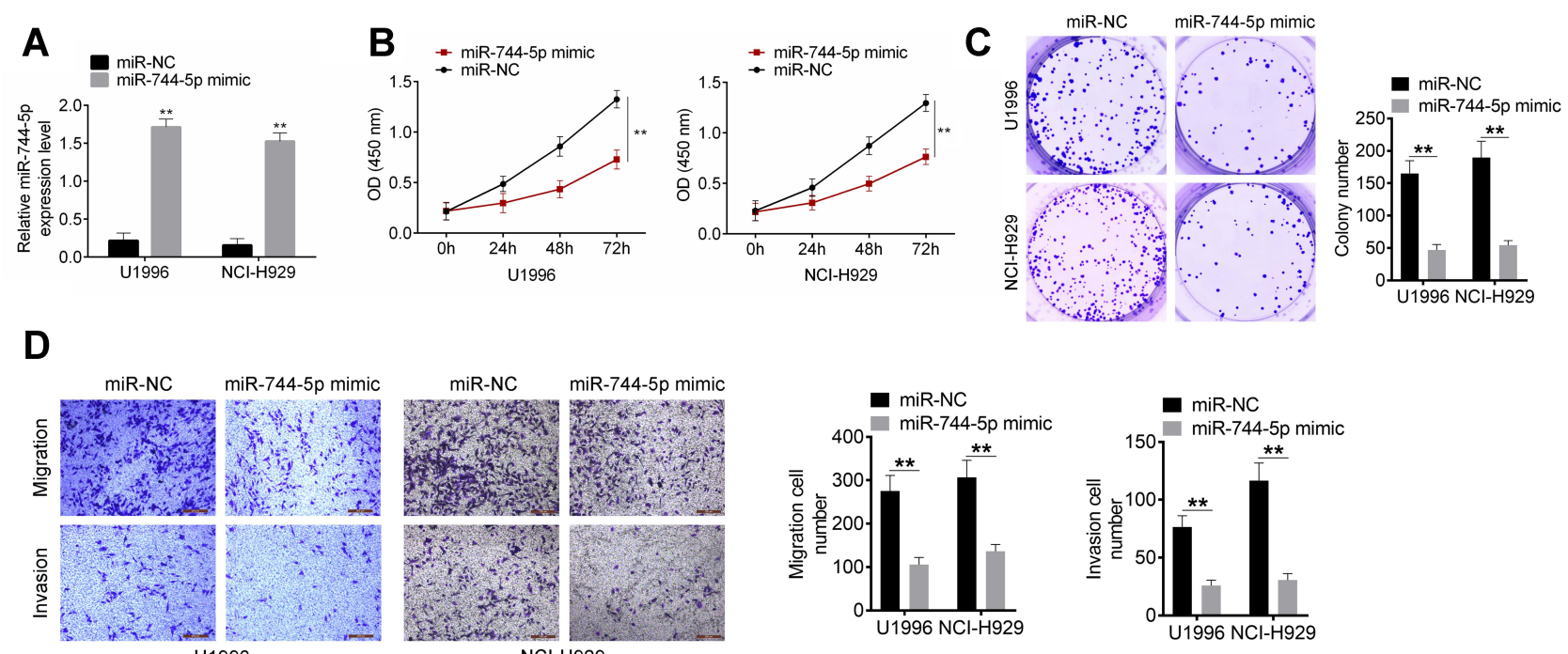

U1996
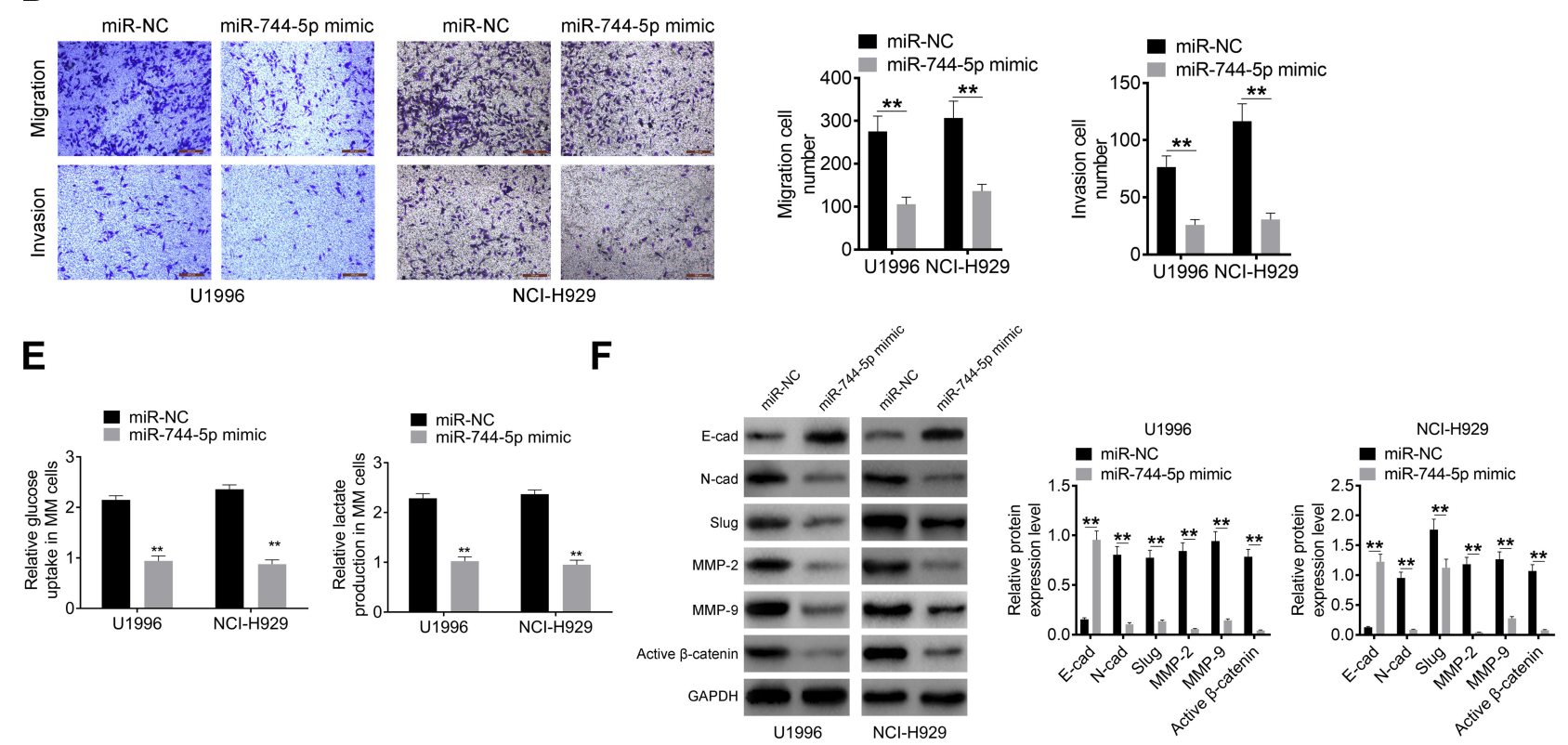

Figure 2 miR-744-5p inhibited MM cells proliferation, invasion, migration, and glycolysis. (A) qRT-PCR revealed that miR-744-5p expression was successfully up-regulated by transfection. miR-744-5p expression was normalized to U6. (B) CCK-8 assay showed prominently lower OD450 value of UI996 and NCl-H929 cells in miR-744-5p mimic group than that in the miR-NC group. (C) Plate clone formation experiment exhibited obviously declined colony number of UI996 and $\mathrm{NCl}-\mathrm{H} 929$ cells in the miR-744-5p mimic group than that in the miR-NC group. (D) Transwell experiment revealed remarkably decreased invasion and migration cell number of miR-744-5p mimic group when compared with miR-NC group. (E) U1996 and NCl-H929 cells of miR-744-5p mimic group exhibited markedly lower glucose uptake and lactate production than that of the miR-NC group. (F) Western blot showed that, significantly higher E-cadherin protein expression and lower N-cadherin, Slug, MMP-2, MMP-9, and active $\beta$-catenin proteins expression was observed in $\mathrm{U} 1996$ and $\mathrm{NCl}-\mathrm{H} 929$ cells of the miR-744-5p mimic group when compared with the miR-NC group. miR-744-5p increased E-cadherin protein expression and decreased the expression of $\mathrm{N}$-cadherin, Slug, MMP-2, MMP-9, and active $\beta$-catenin proteins. Each protein expression was normalized to GAPDH. $* * P<0.01$ when compared with the miR-NC group.

Abbreviations: MM, multiple myeloma; qRT-PCR, quantitative real-time polymerase chain reactions; CCK-8, cell counting kit-8, E-cad, E-cadherin; N-cad, N-cadherin.

NCI-H929 cells of the miR-744-5p mimic group when compared with the miR-NC group $(P<0.01)$ (Figure $2 \mathrm{~F})$.

\section{miR-744-5p Directly Inhibited SOX 12 Expression in MM}

Two online databases, including Targetscan and Starbase, indicated that miR-744-5p was a potential miRNA that targets SOX12. The binding sites were located in the 3 '-UTR area of SOX12 (Figure 3A). Dual-luciferase reporter gene assay was conducted with U1996 cells to confirm SOX12 as a direct target of miR-744-5p. As a result, compared with the miR-NC group, U1996 cells of the miR-744-5p mimic group exhibited prominently lower relative luciferase activity of SOX12-WT luciferase reporter $(P<0.01)$. Meanwhile, markedly higher relative luciferase activity of SOX12-WT luciferase reporter was found in U1996 cells of miR-744-5p inhibitor group when relative to NC-inh group $(P<0.01)$. However, these effects were revived when SOX12-Mut luciferase reporter was cotransfected into U1996 cells (Figure 3B). These data supported that miR-744-5p directly regulated SOX12 via binding to the 3'-UTR area of SOX12. The analyses of RNA pull-down experiment exhibited that only the bio-SOX12-Sense remarkably pulled down the miR-744-5p $(P<0.01)$ (Figure $3 \mathrm{C})$. In vitro studies indicated that U1996 and NCI-H929 cells of the miR-744-5p mimic group had seriously lower SOX12 mRNA than that of the miR-NC group $(P<0.01)$ (Figure 3D). Simultaneously, compared with the miR-NC group, U1996 and NCI-H929 cells of the miR-744-5p mimic group showed much lower SOX12 and active $\beta$-catenin proteins expression 

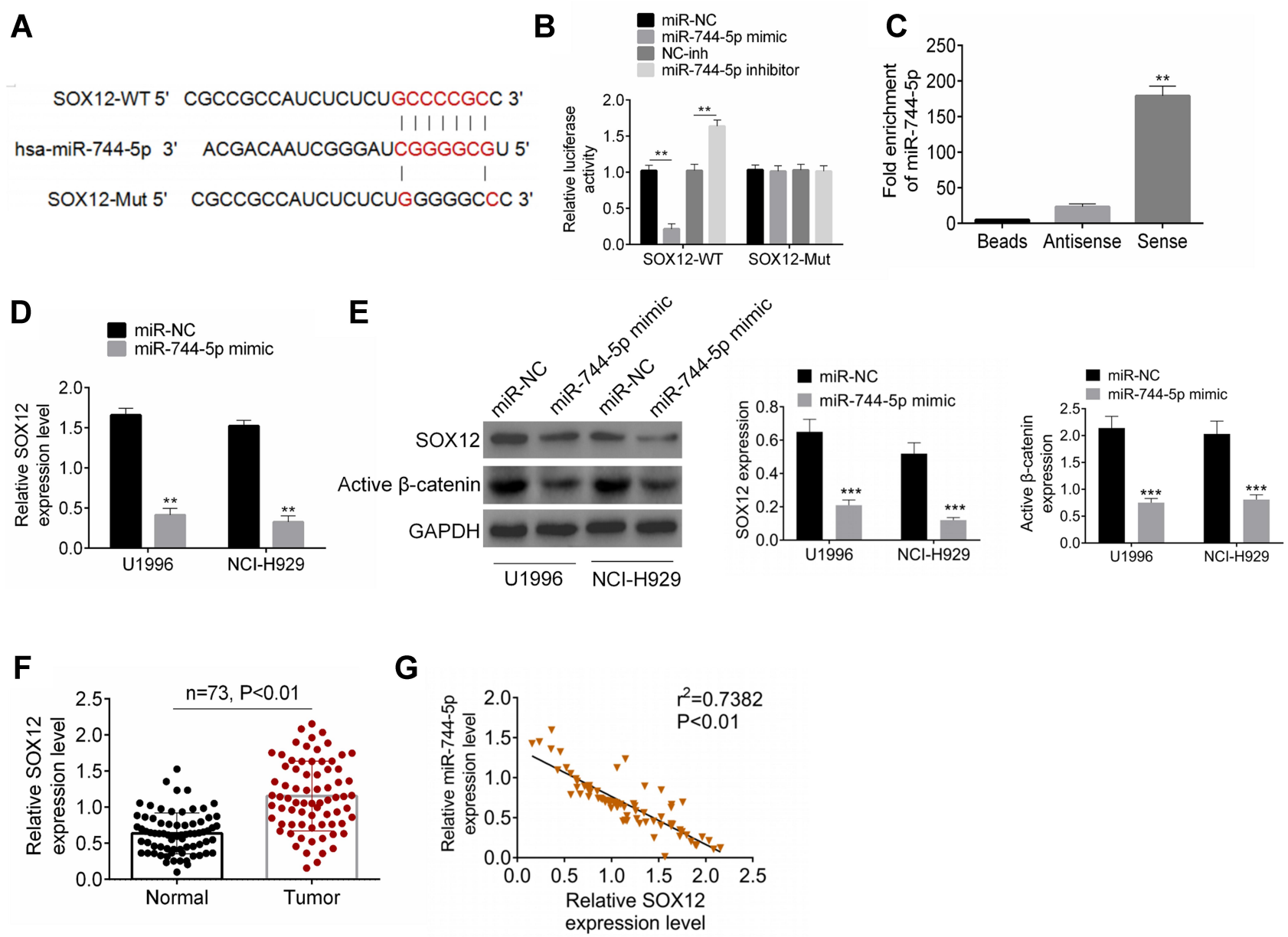

Figure 3 miR-744-5p directly inhibited SOXI2 expression in MM. (A) The binding sites between miR-744-5p and SOXI2 were located in the $3^{\prime}-\mathrm{UTR}$ area of SOXI2. (B) Dual-luciferase reporter gene assay revealed that miR-744-5p directly regulated SOXI2 via binding to the 3 '-UTR area of SOXI2. $* * P<0.01$ when compared with the miRNC group or miR-inh group. (C) RNA pull-down experiment exhibited that miR-744-5p was remarkably pulled down by bio-SOXI2-Sense. **P<0.0I when compared with Beads group and Antisense group. (D) UI996 and NCI-H929 cells of the miR-744-5p mimic group had seriously lower SOXI2 mRNA than that of miR-NC group. SOXI2 mRNA expression was normalized to GAPDH. (E) U1996 and NCl-H929 cells of the miR-744-5p mimic group had much lower SOXI2 and active $\beta$-catenin proteins expression than that of the miR-NC group. SOXI2 and active $\beta$-catenin proteins expression was normalized to GAPDH. $* * P<0.01$ or $* * * P<0.001$ when compared with the miR-NC group. (F) Distinctly higher SOX 12 mRNA expression was shown in bone marrow tissues of MM patients than that in normal bone marrow tissues. SOXI2 expression was normalized to GAPDH. (G) The expression of miR-744-5p and SOXI 2 mRNA was negatively correlated in bone marrow tissues of MM patients. miR-744-5p expression was normalized to $\mathrm{U} 6$ and SOXI2 expression was normalized to GAPDH.

Abbreviation: MM, multiple myeloma.

$(P<0.001)$ (Figure 3E). In addition, distinctly higher SOX12 mRNA expression was shown in bone marrow tissues of MM patients than in normal bone marrow tissues $(P<0.01)$ (Figure $3 F)$. The expression of miR-744-5p and SOX12 mRNA was negatively correlated in bone marrow tissues of MM patients $(P<0.01)$ (Figure 3G). All of these results illustrated that miR$744-5 p$ directly inhibited the expression of SOX12 in MM.

\section{Down-Regulation of miR-744-5p Exacerbated Malignant Phenotype of MM Cells in vitro by Elevating SOXI2}

In vitro rescue experiments were conducted using U1996 cells to verify the mechanism of miR-744-5p and SOX12 in the development of MM. As exhibited in Figure 4A, distinctly higher SOX12 mRNA expression was observed in U1996 cells of the miR-744-5p inhibitor group when relative to the $\mathrm{NC}$-inh group $(P<0.01)$. However, relative to the miR-744-5p inhibitor group, the expression of SOX12 mRNA in U1996 cells of miR-744-5p inhibitor + siSOX12 group was significantly reduced $(P<0.01)$. Therefore, SOX12 expression was elevated by inhibition of miR-744-5p. Subsequently, the malignant phenotype of U1996 cells was studied. CCK-8 assay and plate clone formation experiment showed dramatically higher OD450 value and colony number in miR-744-5p inhibitor group when compared with NC-inh group $(P<0.01)$. Conversely, 
miR-744-5p inhibitor + siSOX12 group exhibited seriously lower OD450 value and colony number than that of the miR-744-5p inhibitor group $(P<0.01)$ (Figure $4 \mathrm{~B}$ and C). Based on data from Transwell experiment, U1996 cells of the miR-744-5p inhibitor group had obviously more invasion and migration cells than the NC-inh group $(P<0.01)$, whereas U1996 cells of miR-744-5p inhibitor + siSOX12 group exhibited much less invasion and migration cells than the miR-744-5p inhibitor group $(P<0.01)$ (Figure 4D). Furthermore, much higher relative glucose uptake and lactate production was found in the U1996 cells of the miR-744-5p inhibitor group relative to the NCinh group $(P<0.01)$. However, in comparison with the
miR-744-5p inhibitor group, the relative glucose uptake and lactate production of U1996 cells in the miR-744-5p inhibitor + siSOX12 group was prominently declined $(P<0.01)$ (Figure 4E). Analysis of Western blot exhibited that U1996 cells of the miR-744-5p inhibitor group had markedly lower E-cadherin protein expression and higher N-cadherin, Slug, MMP-2, MMP-9, and active $\beta$-catenin proteins expression that $\mathrm{NC}$-inh group $(P<0.01)$. On the opposite, relative to the miR-744-5p inhibitor group, seriously higher E-cadherin protein expression and lower N-cadherin, Slug, MMP-2, MMP-9, and active $\beta$-catenin proteins expression was observed in the miR-744-5p inhibitor + siSOX12 group $(P<0.01)$ (Figure 4F).
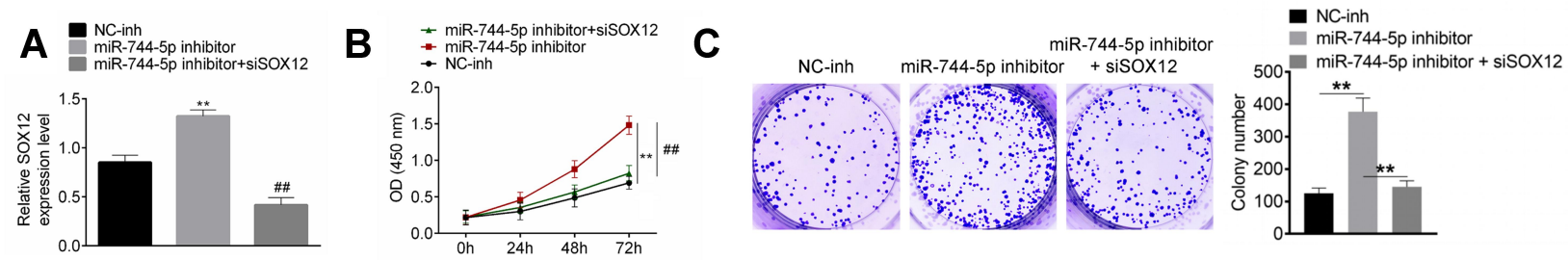

D
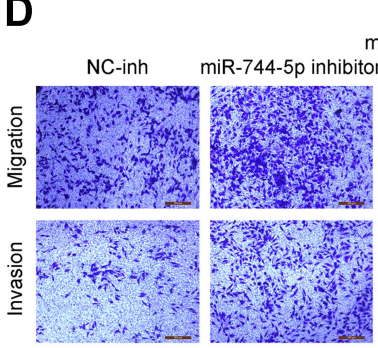

miR-744-5p inhibitor

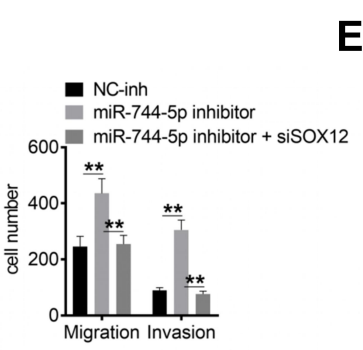

E
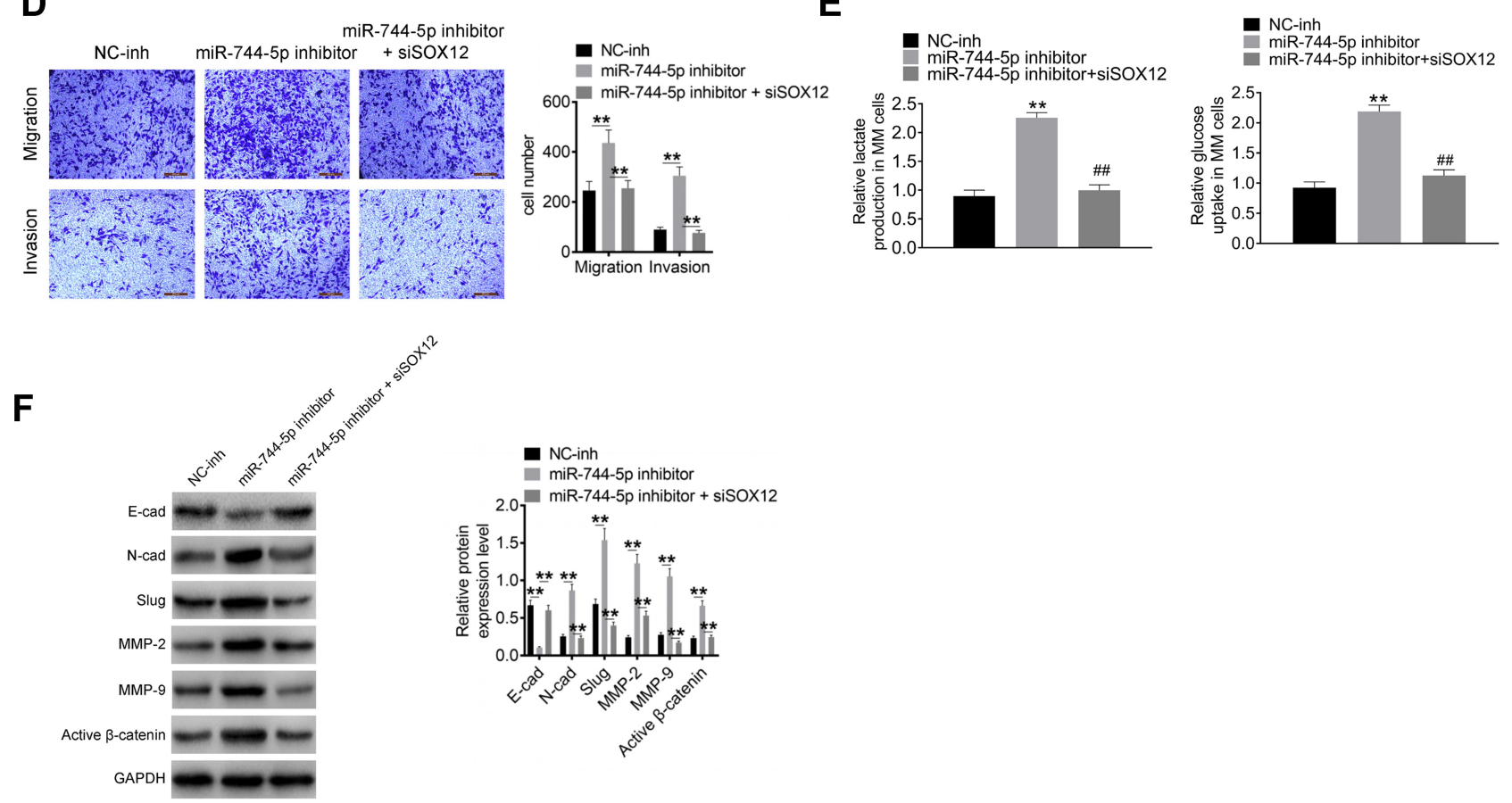

Figure 4 Down-regulation of miR-744-5p exacerbated malignant phenotype of MM cells in vitro by elevating SOXI2. (A) SOXI2 expression in UI996 cells was successfully regulated by transfection. ${ }^{*} P<0.01$ when compared with the NC-inh group. SOXI2 expression was normalized to GAPDH. ${ }^{\#} P<0.01$ when compared with the miR-744-5P inhibitor group. (B) CCK-8 assay indicated that down-regulation of miR-744-5p enhanced UI996 cells proliferation by elevating SOXI2. $* * P<0.01$ when compared with the NC-inh group. ${ }^{\#} P<0.01$ when compared with the miR-744-5p inhibitor group. (C) Plate clone formation experiment revealed that down-regulation of miR-744-5p enhanced UI996 cells malignant proliferation by elevating SOXI2. **P<0.0I. (D) Transwell experiment illustrated that down-regulation of miR-744-5p intensified UI996 cells invasion and migration via elevating SOXI2. ${ }^{*} P<0.0 \mathrm{I}$. (E) Down-regulation of miR-744-5p increased glucose uptake and lactate production of UI996 cells through elevating SOXI2. **P $<0.01$ when compared with the NC-inh group. ${ }^{\#} P<0.01$ when compared with the miR-744-5p inhibitor group. (F) Western blot exhibited that, relative to the miR-744$5 p$ inhibitor group, much higher E-cadherin protein expression and lower N-cadherin, Slug, MMP-2, MMP-9, and active $\beta$-catenin proteins expression was observed in the miR-744-5p inhibitor + siSOX 12 group. Down-regulation of miR-744-5p reduced E-cadherin protein expression and increased the expression of N-cadherin, Slug, MMP-2, MMP-9, and active $\beta$-catenin proteins in U1996 cells through elevating SOXI2. Each protein expression was normalized to GAPDH. $* * P<0.01$.

Abbreviations: MM, multiple myeloma; CCK-8, cell counting kit-8. 


\section{miR-744-5p Inhibited the Growth of MM Xenograft Tumors in vivo}

The expression of miR-744-5p and SOX12 mRNA in MM xenograft tumors were detected by qRT-PCR. As a result, markedly higher miR-744-5p expression and lower SOX12 mRNA expression were noticed in MM xenograft tumors of the miR-744-5p mimic group when relative to the miR-NC group $(P<0.001)$ (Figure 5A). Moreover, dramatically lower xenograft tumor volume was observed in the miR-744-5p mimic group in comparison with the miRNC group $(P<0.001)$ (Figure 5B). All xenograft tumors were stripped after nude mice were sacrificed (Figure 5C). The weight of all xenograft tumors was measured. Obviously, compared with the miR-NC group, the weight of xenograft tumors in the miR-744-5p mimic group was prominently reduced $(P<0.001)$ (Figure 5D).

\section{Discussion}

The molecular mechanism exploration of MM progression is conducive to find new strategies for the target treatment of this annoying disease. miRNAs have been extensively explored in many types of human tumors, but relatively few studies have reported the impact of miRNAs on the development of MM. Currently, it has been discovered that
miR-144-3p expression was reduced in MM cell lines. MM cells proliferation and colony formation was suppressed by transfecting miR-144-3p mimic into MM cells. ${ }^{12}$ Besides, miR-29b, miR-125a, miR-320c, miR-342, and miR-363 were identified as potential tumor suppressors in $\mathrm{MM}^{13-15}$ By contrast, miR-181, miR-340-5p, miR-9, miR-19b, and miR-20a were aberrantly up-regulated in MM. They promoted the malignant development of MM and thus acted as oncogenes in MM. ${ }^{16-19}$ Kubiczkova et $\mathrm{al}^{20}$ reported that miR-744 expression was down-regulated in the peripheral blood of MM patients and miR-744 level could be used as a marker for MM patients survival. Furthermore, $\mathrm{Xu}$ et $\mathrm{al}^{21}$ also researched that miR-744 was down-regulated in MM patients. The decreased miR-744 expression was associated with poor prognosis of MM patients. But unfortunately, the above two studies only reported that the down-regulation of miR-744 predicted the poor prognosis of MM. They did not conduct in-depth studies to explore the molecular mechanisms of miR-744 in regulating MM development. In addition, more data on miR-744 in regulating MM development are not yet available at present. In this research, we proved that the down-regulated miR-744-5p in MM was associated with the low survival rate of MM patients. More importantly, through in vitro and in vivo experiments, this research fully
A

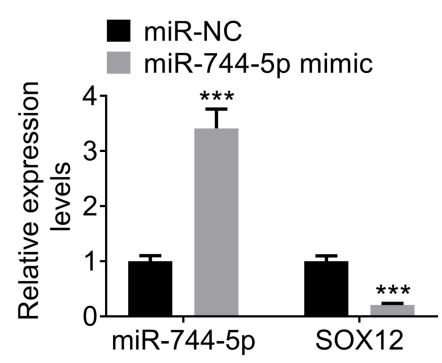

C

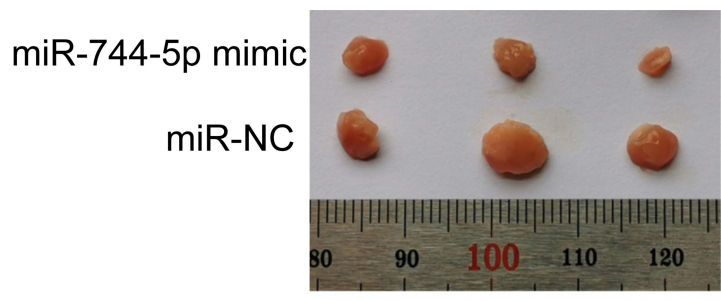

B

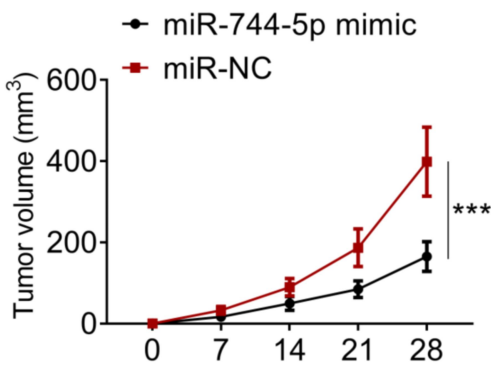

D

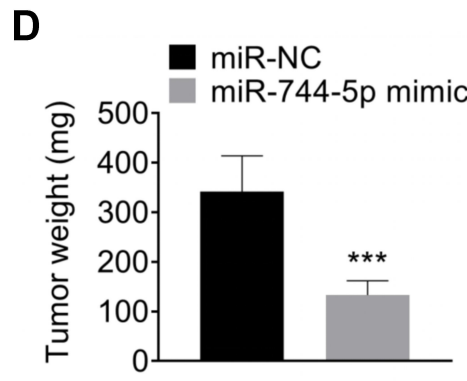

Figure 5 miR-744-5p inhibited the growth of MM xenograft tumors in vivo. (A) Markedly higher miR-744-5p expression and lower SOX 12 mRNA expression was noticed in $M M$ xenograft tumors of the miR-744-5p mimic group when relative to the miR-NC group. miR-744-5p expression was normalized to U6 and SOXI2 expression was normalized to GAPDH. (B) Dramatically lower xenograft tumor volume was observed in the miR-744-5p mimic group in comparison with the miR-NC group. (C) All xenograft tumors were stripped after nude mice were sacrificed. (D) Compared with the miR-NC group, the weight of xenograft tumors in the miR-744-5p mimic group was prominently reduced. $* * * P<0.001$ when compared with the miR-NC group.

Abbreviation: MM, multiple myeloma. 
confirmed that the down-regulated miR-744-5p promoted the malignant development of MM. Simultaneously, this paper initially discovered that miR-744-5p weakened MM malignant development via targeting SOX12/Wnt/ $\beta$-catenin. These findings provided a more precise theoretical basis for the treatment of MM with miR-744-5p as the target.

The function of miRNAs in regulating multiple physiological and pathological processes is mainly achieved by target genes. ${ }^{22}$ Thus, the identification of target genes of miRNAs is one of the primary issues for researching the mechanism. A previous report indicated that SOX12 exacerbated the development of MM via promoting the $\mathrm{Wnt} / \beta$-catenin signaling. ${ }^{4}$ Importantly, Zhang et al ${ }^{23}$ discovered that SOX12 is a target of miR-744. Based on this finding, this research explored the interaction of miR-744-5p and SOX12 in regulating MM. Through dual-luciferase reporter gene assay and RNA pulldown experiment, we further confirmed that SOX12 expression was directly suppressed by miR-744-5p.

In general, SOX12 is considered as a cancerpromoting gene in humans. It has been reported that SOX12 could promote the metastasis of gastric cancer, hepatocellular carcinoma, and also enhance the proliferation and metastasis of colorectal cancer. ${ }^{24-26}$ Moreover, high SOX12 expression was associated with poor overall survival of clear cell renal cell carcinoma cases and lung adenocarcinoma cases. ${ }^{27,28}$ Meanwhile, the proliferation, migration, and invasion of breast cancer cells was significantly weakened after silencing of SOX $12 .{ }^{29}$ In hematologic malignancy, knockdown of SOX12 decreased the colony number of acute myeloid leukemia cells. By knockdown of SOX12, $\beta$-catenin expression was distinctly diminished. Therefore, research suggested that SOX12 might facilitate the progression of acute myeloid leukemia via enhancing $\beta$-catenin expression. ${ }^{30}$ Currently, a study indicated that SOX12 was an oncogene in MM. It aggravated $\mathrm{MM}$ progression via down-regulating $\beta$ catenin expression. ${ }^{4}$ Similar results were found in this research. $\beta$-catenin is a critical downstream effector of the Wnt signaling pathway. It is responsible for cells proliferation and differentiation. Lots of articles have demonstrated that the enhancement of $\mathrm{Wnt} / \beta$-catenin pathway participates in the deterioration of various malignant tumors. ${ }^{31,32}$ In MM, the silencing of $\beta$-catenin elevated the apoptosis ratio and reduced proliferation of $\mathrm{MM}$ cells. ${ }^{33,34}$ Overall, in this paper, miR-744-5p inhibited MM development via inhibiting the SOX12/Wnt/ $\beta$ catenin pathway.
In this study, miR-744-5p up-regulation elevated E-cadherin protein expression, but also reduced N-cadherin, Slug, MMP-2, and MMP-9 proteins expression in MM cells. The opposite results were found by down-regulating miR-744-5p expression. Interestingly, rescue experiments indicated that SOX12 silencing reversed the inhibition effect of miR-744-5p on EMT in MM cells. It is well known that E-cadherin protein is an inhibitor of EMT. N-cadherin, Slug, MMP-2, and MMP-9 proteins are responsible for the enhancement of EMT. Hence, miR-744-5p inhibited MM cells migration and invasion by weakening EMT via reducing SOX12. Moreover, miR-744-5p reduced the glycolysis in MM cells by reducing glucose uptake and lactate production through targeting SOX12. The Warburg effect is considered to be a hallmark of tumors, which is an important feature of glucose metabolism in malignant tumor cells. In the process of the Warburg effect, tumor cells provide energy for themselves through using aerobic glycolysis, and then provide material raw materials for cell synthesis and metabolism by using intermediate products of glycolysis. This process exacerbates the progression of cells. ${ }^{35}$ At last but not the least, animal in vivo experiments were performed in this study. Clearly, miR-744-5p up-regulation distinctly reduced MM cells growth in vivo. This discovery provided a more reliable theoretical basis for $\mathrm{MM}$ therapy with miR-744-5p as the target.

Collectively, this article reported the expression and function of miR-744-5p in MM. Results indicated that miR-744-5p expression was declined in MM patients and cells. miR-744-5p up-regulation prominently inhibited MM cells malignant development in vitro and in vivo. The mechanism might be that miR-744-5p suppressed the SOX12/Wnt/ß-catenin pathway in MM cells. Hence, miR$744-5 p$ was suggested as a potential therapeutic target for MM.

\section{Highlights}

(1) The expression of miR-744-5p was reduced in MM patients and cells.

(2) miR-744-5p inhibited MM cells proliferation, invasion, migration, and glycolysis.

(3) miR-744-5p directly inhibited SOX12 expression in MM.

(4) miR-744-5p silencing promoted MM cells malignant phenotype by elevating SOX12.

(5) miR-744-5p inhibited the growth of MM xenograft tumors in vivo. 


\section{Acknowledgment}

Bingling Guo and Chunyan Xiao are co-first authors.

\section{Disclosure}

The authors report no conflicts of interest for this work.

\section{References}

1. Liu S, Zhang Y, Huang C, et al. miR-215-5p is an anticancer gene in multiple myeloma by targeting RUNX1 and deactivating the PI3K/ AKT/mTOR pathway. J Cell Biochem. 2020;121(2):1475-1490.

2. Chen R, Zhang X, Wang C. LncRNA HOXB-AS1 promotes cell growth in multiple myeloma via FUT4 mRNA stability by ELAVL1. J Cell Biochem. 2019.

3. Yang X, Huang H, Wang X, et al. Knockdown of lncRNA SNHG16 suppresses multiple myeloma cell proliferation by sponging miR-342-3p. Cancer Cell Int. 2020;20(1):38.

4. Gao Y, Li L, Hou L, et al. SOX12 promotes the growth of multiple myeloma cells by enhancing Wnt/ $\beta$-catenin signaling. Exp Cell Res. 2020;388(1):111814. doi:10.1016/j.yexcr.2020.111814

5. Ye J, Xu M, Tian X, et al. Research advances in the detection of miRNA. J Pharm Anal. 2019;9(4):217-226.

6. Liu XK, Chen D, Li X. MiR-335 suppresses cell proliferation and migration by upregulating CRKL in bladder cancer. Eur Rev Med Pharmacol Sci. 2019;23(6):2399-2408.

7. Liu G, Li B, et al. Role of miRNA in transformation from normal tissue to colorectal adenoma and cancer. J Cancer Res Ther. 2019;15 (2):278.

8. Kleemann M, Schneider H, Unger K, et al. MiR-744-5p inducing cell death by directly targeting HNRNPC and NFIX in ovarian cancer cells. Sci Rep. 2018;8(1):9020. doi:10.1038/s41598-018-27438-6

9. Chen S, Shi F, Zhang W, et al. miR-744-5p inhibits non-small cell lung cancer proliferation and invasion by directly targeting PAX2. Technol Cancer Res Treat. 2019;18:1533033819876913.

10. Sui Y, Lin G, Zheng Y, et al. LncRNA MAFG-AS1 boosts the proliferation of lung adenocarcinoma cells via regulating miR-7445p/MAFG axis. Eur J Pharmacol. 2019;859:172465. doi:10.1016/j. ejphar.2019.172465

11. Liang Z, Wang X, Xu X, et al. MicroRNA-608 inhibits proliferation of bladder cancer via AKT/FOXO3a signaling pathway. Mol Cancer. 2017;16(1):96. doi:10.1186/s12943-017-0664-1

12. Zhao Y, Xie Z, Lin J, et al. MiR-144-3p inhibits cell proliferation and induces apoptosis in multiple myeloma by targeting c-Met. Am J Transl Res. 2017;9(5):2437-2446.

13. Alzrigat M, Jernberg-Wiklund H. The miR-125a and miR-320c are potential tumor suppressor microRNAs epigenetically silenced by the polycomb repressive complex 2 in multiple myeloma. $R N A$ Dis. 2017;4(2):e1529.

14. Gowda PS, Wildman BJ, Trotter TN, et al. Runx2 suppression by miR-342 and miR-363 inhibits multiple myeloma progression. Mol Cancer Res. 2018;16(7):1138-1148. doi:10.1158/1541-7786.MCR17-0606

15. Wang H, Ding Q, Wang M, et al. miR-29b inhibits the progression of multiple myeloma through downregulating FOXP1. Hematology. 2019;24(1):32-38. doi:10.1080/10245332.2018.1502961

16. Yuan R, Liu N, Yang J, et al. The expression and role of miR-181a in multiple myeloma. Medicine (Baltimore). 2018;97(35):e12081. doi:10.1097/MD.0000000000012081

17. Li Z, Wong KY, Calin GA, et al. Epigenetic silencing of miR-340-5p in multiple myeloma: mechanisms and prognostic impact. Clin Epigenetics. 2019;11(1):71. doi:10.1186/s13148-019-0669-2
18. Huang G, Liu X, Zhao X, et al. MiR-9 promotes multiple myeloma progression by regulating TRIM56/NF- $\kappa$ B pathway. Cell Biol Int. 2019;43(11):1223-1233. doi:10.1002/cbin.11104

19. Yuan J, Su Z, Gu W, et al. MiR-19b and miR-20a suppress apoptosis, promote proliferation and induce tumorigenicity of multiple myeloma cells by targeting PTEN. Cancer Biomarkers. 2019;24(3):279-289. doi:10.3233/CBM-182182

20. Kubiczkova L, Kryukov F, Slaby O, et al. Circulating serum microRNAs as novel diagnostic and prognostic biomarkers for multiple myeloma and monoclonal gammopathy of undetermined significance. Haematologica. 2014;99(3):511-518. doi:10.3324/ haematol.2013.093500

21. Xu P, Xia T, Ling Y, et al. MiRNAs with prognostic significance in multiple myeloma: a systemic review and meta-analysis. Medicine (Baltimore). 2019;98(33):e16711. doi:10.1097/MD.0000000000016711

22. Ziebarth JD, Bhattacharya A, Cui Y. Functional analysis of genetic variants and somatic mutations impacting microRNA-target recognition: bioinformatics resources. Methods Mol Biol. 2019;101-120.

23. Zhang W, Liu K, Liu S, et al. MicroRNA-744 inhibits migration and invasion of hepatocellular carcinoma cells by targeting SOX12. Oncol Rep. 2018;40(6):3585-3592.

24. Du F, Feng W, Chen S, et al. Sex determining region Y-box 12 (SOX12) promotes gastric cancer metastasis by upregulating MMP7 and IGF1. Cancer Lett. 2019;452:103-118. doi:10.1016/j. canlet.2019.03.035

25. Du F, Chen J, Liu H, et al. SOX12 promotes colorectal cancer cell proliferation and metastasis by regulating asparagine synthesis. Cell Death Dis. 2019;10(3):239. doi:10.1038/s41419-019-1481-9

26. Huang W, Chen Z, Shang X, et al. Sox12, a direct target of FoxQ1, promotes hepatocellular carcinoma metastasis through up-regulating twist1 and FGFBP1. Hepatology. 2015;61(6):1920-1933. doi:10.1002/hep.27756

27. Gu W, Wang B, Wan F, et al. SOX2 and SOX12 are predictive of prognosis in patients with clear cell renal cell carcinoma. Oncol Lett. 2018;15(4):4564-4570.

28. Li L, Zhang T, Chen Y, et al. [Expression of transcription factor SOX12 in lung adenocarcinoma and its clinical significance]. Nan Fang Yi Ke Da Xue Xue Bao. 2019;39(2):186-191. Chinese.

29. Ding H, Quan H, Yan W, et al. Silencing of SOX12 by shRNA suppresses migration, invasion and proliferation of breast cancer cells. Biosci Rep. 2016;36(5):5. doi:10.1042/BSR20160053

30. Wan H, Cai J, Chen F, et al. SOX12: a novel potential target for acute myeloid leukaemia. $\mathrm{Br} \quad J$ Haematol. 2017;176(3):421-430. doi:10.1111/bjh.14425

31. Vilchez V, Turcios L, Marti F,et al. Targeting Wnt/ $\beta$-catenin pathway in hepatocellular carcinoma treatment. World $J$ Gastroenterol. 2016;22(2):823-832. doi:10.3748/wjg.v22.i2.823

32. Gao L, Chen B, Li J, et al. Wnt/ $\beta$-catenin signaling pathway inhibits the proliferation and apoptosis of U87 glioma cells via different mechanisms. PLoS One. 2017;12(8):e0181346. doi:10.1371/journal. pone.0181346

33. Su N, Wang P, Li Y. Role of Wnt/ $\beta$-catenin pathway in inducing autophagy and apoptosis in multiple myeloma cells. Oncol Lett. 2016;12(6):4623-4629. doi:10.3892/ol.2016.5289

34. Zhang JG, Li YP, Fang ZQ, et al. [Effect of siRNA-interfering $\beta$ catenin expression on MDR of human multiple myeloma cell line]. Zhongguo Shi Yan Xue Ye Xue Za Zhi. 2019;27(2):477-481. Chinese.

35. Zhu W, Huang Y, Pan Q, et al. MicroRNA-98 suppress warburg effect by targeting HK2 in colon cancer cells. Dig Dis Sci. 2016;62 (3):1-9. 


\section{Publish your work in this journal}

OncoTargets and Therapy is an international, peer-reviewed, open access journal focusing on the pathological basis of all cancers, potential targets for therapy and treatment protocols employed to improve the management of cancer patients. The journal also focuses on the impact of management programs and new therapeutic agents and protocols on patient perspectives such as quality of life, adherence and satisfaction. The manuscript management system is completely online and includes a very quick and fair peer-review system, which is all easy to use. Visit http://www.dovepress.com/ testimonials.php to read real quotes from published authors.

Submit your manuscript here: https://www.dovepress.com/oncotargets-and-therapy-journal 